\title{
Independent DSG4 frameshift variants in cats with hair shaft dystrophy
}

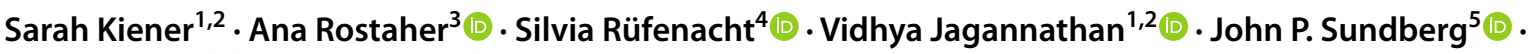 \\ Monika Welle ${ }^{2,6}$ (D) Tosso Leeb ${ }^{1,2}$ (D)
}

Received: 22 October 2021 / Accepted: 25 November 2021 / Published online: 8 December 2021

(c) The Author(s) 2021

\begin{abstract}
Investigations of hereditary phenotypes in spontaneous mutants may help to better understand the physiological functions of the altered genes. We investigated two unrelated domestic shorthair cats with bulbous swellings of the hair shafts. The clinical, histopathological, and ultrastructural features were similar to those in mice with lanceolate hair phenotype caused by loss-of-function variants in $D s g 4$ encoding desmoglein 4 . We sequenced the genomes from both affected cats and compared the data of each affected cat to 61 control genomes. A search for private homozygous variants in the DSG4 candidate gene revealed independent frameshift variants in each case, c.76del or p.Ile26fsLeu*4 in case no. 1 and c.1777del or p.His593Thrfs*23 in case no. 2. DSG4 is a transmembrane glycoprotein located primarily in the extracellular part of desmosomes, a complex of adhesion molecules responsible for connecting the keratin intermediate filaments of neighbouring epithelial cells. Desmosomes are essential for normal hair shaft formation. Both identified DSG4 variants in the affected cats lead to premature stop codons and truncate major parts of the open-reading frame. We assume that this leads to a complete loss of DSG4 function, resulting in an incorrect formation of the desmosomes and causing the development of defective hair shafts. Together with the knowledge on the effects of DSG4 variants in other species, our data suggest that the identified $D S G 4$ variants cause the hair shaft dystrophy. To the best of our knowledge, this study represents the first report of pathogenic $D S G 4$ variants in domestic animals.
\end{abstract}

Keywords Felis catus · Whole-genome sequencing · Dermatology · Genodermatosis · Alopecia $\cdot$ Lanceolate $\cdot$ Hair shaft dysplasia

Communicated by Joan Cerdá.

Tosso Leeb

tosso.leeb@vetsuisse.unibe.ch

Sarah Kiener

sarah.kiener@vetsuisse.unibe.ch

Ana Rostaher

arostaher@vetclinics.uzh.ch

Silvia Rüfenacht

s.ruefenacht@dermavet.ch

Vidhya Jagannathan

vidhya.jagannathan@vetsuisse.unibe.ch

John P. Sundberg

john.sundberg@jax.org

Monika Welle

monika.welle@vetsuisse.unibe.ch
1 Institute of Genetics, Vetsuisse Faculty, University of Bern, 3001 Bern, Switzerland

2 University of Bern, 3001 Bern, Switzerland

3 Clinic for Small Animal Internal Medicine, Vetsuisse Faculty, University of Zurich, 8057 Zurich, Switzerland

4 DermaVet, Tierklinik Aarau West, 5036 Oberentfelden, Switzerland

5 The Jackson Laboratory, Bar Harbor, ME 04609, USA

6 Institute of Animal Pathology, Vetsuisse Faculty, University of Bern, 3001 Bern, Switzerland 


\section{Introduction}

Hair is composed of terminally differentiated dead keratinocytes. It is characteristic for terrestrial mammals, and involved in various functions such as physical protection, thermal regulation, or sensory perception. The hair follicle, a complex miniorgan residing in the dermal layer of the skin, is responsible for the formation of hair (Schneider et al. 2009). The hair shaft consists of the hair cuticle, the cortex, and the innermost medulla. It is surrounded by the inner root sheath, the companion layer, and the outer root sheath (Cheng and Bayliss 2008; Shimomura and Christiano 2010; Welle and Wiener 2016).

Significant progress has been made in identifying numerous genes expressed in the hair follicle (Shimomura and Christiano 2010; Wiener et al. 2020). A malfunctioning hair follicle, e.g., due to a genetic defect, represents one of several possible causes for hair loss resulting in alopecia or hypotrichosis (Cheng and Bayliss 2008; Schneider et al. 2009; Shimomura and Christiano 2010; Welle and Wiener 2016; Ahmed et al. 2019).

Targeted or spontaneous mutant mice provided many valuable resources for dermatological research (Sundberg 1994; Sundberg and King 1996, 2000; Nakamura et al. 2001; Chen et al. 2008). An ethylnitrosourea induced and, subsequently, a spontaneous mouse mutant with irregular hair shafts characterized by focal deformities and pronounced enlargement at the apex, resembling a lance head, were termed lanceolate hair (lah and lah-J) (Montagutelli et al. 1996; Sundberg et al. 2000). The causative genetic variant for the lah mouse mutant was identified in the $D s g 4$ gene encoding desmoglein 4 (Kljuic et al. 2003). Several additional spontaneous allelic mutants with the same phenotype have been discovered in mice (Berry and Sundberg, unpublished data). A related phenotype in humans, localized autosomal recessive hypotrichosis (LAH, OMIM \#607903), is due to genetic variants in the human ortholog, DSG4 (Kljuic et al. 2003). Finally, a spontaneous lanceolate hair phenotype due to a $D s g 4$ variant also exists in rats (Jahoda et al. 2004).

We previously described the clinical and histopathological findings in a litter of cats with alopecia and hair shaft irregularities that closely resembled the phenotype of $D s g 4$-deficient lah mice (Rostaher et al. 2021). In the present study, we investigated one additional unrelated case of a similar hair shaft dystrophy and searched for the underlying causative genetic variants in the previously reported cats.

\section{Methods}

\section{Animal selection}

This study included two unrelated domestic shorthair (DSH) cats affected by hair shaft dystrophy, and 61 genetically diverse control cats. The 63 sequenced cats included 34 purebred cats from 11 different breeds and 29 randombred domestic cats (Table S1). Genomic DNA was isolated from ETDA blood samples with the Maxwell RSC Whole Blood Kit using a Maxwell RSC instrument (Promega, Dübendorf, Switzerland).

\section{Histopathological examinations}

Skin biopsies from alopecic skin were taken under general anesthesia according to standard procedures. The biopsies were processed routinely and stained with hematoxylin and eosin, prior to histological evaluation.

\section{Whole-genome sequencing}

Illumina TruSeq PCR-free DNA libraries with $\sim 300 \mathrm{bp}$ insert size of both affected cats were prepared and sequenced at $24 \times$ coverage (case no. 1 ) and $21 \times$ coverage (case no. 2) on a NovaSeq 6000 instrument. The reads were mapped to the FelCat9.0 cat reference genome assembly as described (Jagannathan et al. 2019). The sequence data were submitted to the European Nucleotide Archive with the study accession PRJEB7401 and sample accessions SAMEA7853384 (case no. 1) and SAMEA7853385 (case no. 2).

\section{Variant calling and filtering}

Variant calling was performed as described (Jagannathan et al. 2019). The SnpEff software was used together with NCBI annotation release 104 of the FelCat9.0 genome reference assembly to predict the functional effects of the called variants (Cingolani et al. 2012). For variant filtering, we used 61 control genomes (Table S1). Filtering was done with a self-written $\mathrm{C}++$ program on a modified vcffile containing genotype calls of the 2 affected cats and the 61 control cats, and SnpEff predicted functional effects of all variants. 


\section{Gene analysis}

Numbering within the feline DSG4 gene corresponds to the NCBI RefSeq accession numbers XM_019815116.1 (mRNA) and XP_019670675.1 (protein).

\section{Sanger sequencing}

Sanger sequencing of PCR amplicons was used to confirm the candidate variants $D S G 4:$ c.76del and $D S G 4: c .1777 \mathrm{del}$ and to genotype cats. We used forward and reverse primers together with the AmpliTaqGold360Mastermix (Thermo Fisher Scientific, Waltham, MA, USA) to amplify a PCR product from genomic DNA (Table 1). After treatment with shrimp alkaline phosphatase and exonuclease I, PCR amplicons were sequenced on an ABI 3730 DNA Analyzer (Thermo Fisher Scientific). The Sequencher 5.1 software was used to analyze the Sanger sequences (GeneCodes, Ann Arbor, MI, USA).

\section{Results}

\section{Family anamnesis, clinical examinations, and histopathology}

Case no. 1 was a female random-bred DSH cat, born in 2019 on a farm in Switzerland. An unusual generalized moderate-to-severe hypotrichosis was observed at the first check-up examination at 3 months of age. The cat was otherwise in good general health. The owner reported that a maternal half-sibling of this cat had been born naked and died within a few days after birth. The hair loss in case no. 1 symmetrically affected the convex pinnae, parts of the face, the back, and the legs including the dorsal paws (Fig. 1a, b). The cat was mildly pruritic. Dermatophytosis and infestation with ectoparasites as underlying cause for the alopecia were excluded clinically and by treatment with Fluralaner (Bravecto(R), MSD Animal Health GmbH, Switzerland) and topical enilconazole (Imaverol(R), Covetrus AG, Switzerland). The trichogram revealed broken and split hair shafts. The hair shafts presented with severe
Table 1 Details of the primers used for Sanger sequencing

\begin{tabular}{lllll}
\hline Variant & Primer name & Primer sequence $\left(5^{\prime}\right.$ to $\left.3^{\prime}\right)$ & $\begin{array}{l}\text { Amplicon } \\
\text { length }(\mathrm{bp})\end{array}$ & $T_{M}\left({ }^{\circ} \mathrm{C}\right)$ \\
\hline DSG4:c.76del & Primer F1 & GGAGGGCAAAGAGCCTGTAT & 362 & 60 \\
& Primer R1 & TGGGTTTGCCATTGCTATTT & & \\
DSG4:c.1777del & Primer F2 & GACGGCTGAGCAGCTTTTAT & 445 & 60 \\
& Primer R2 & GCCCTTATTAGCCCCATTGT & & \\
\hline
\end{tabular}

Fig. 1 Clinical phenotype of the affected cats with hypotrichosis and alopecia on the convex pinnae, parts of the face, the back, and the legs. a, b Case no. 1. c, d Case no. 2
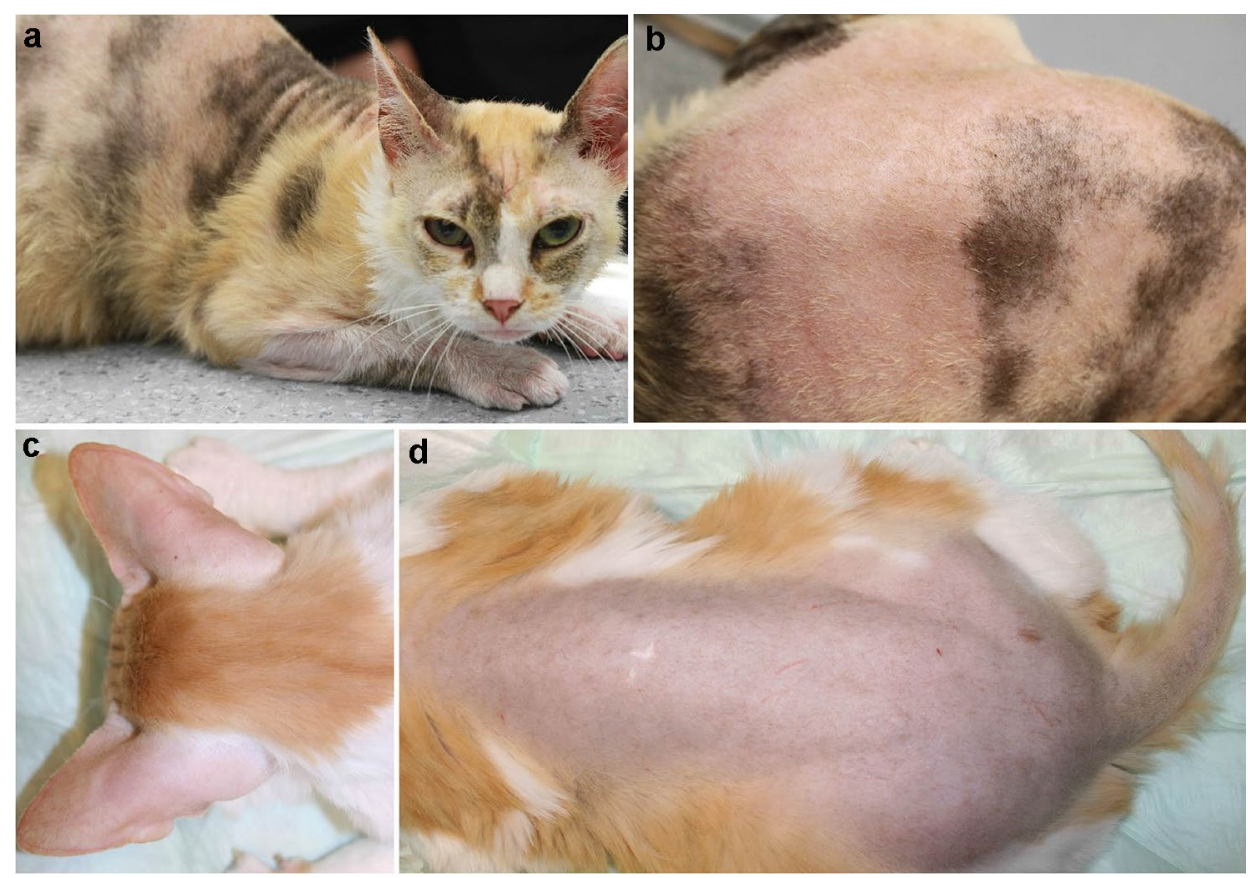
Fig. 2 Clinical and histological features of the lanceolate hair shafts and follicles of cases no. 1 and no. 2 (a-e). a The Dermascope image shows hair with irregular thickenings of the hair shafts (red circle). b-c Trichogram of hair with lancehead shaped distal ends. $\mathbf{d}$ The histological image of an anagen hair follicle shows a bulbous or lance-head shaped swelling of the already fully cornified hair shaft (black arrow) just distal to the melanogenic zone at the border of inferior portion and isthmus. This is associated with individual cells or small cell clusters in the precortex and premedulla (white arrow) that are enlarged, rounded, and have abundant pale cytoplasm, suggesting that they are undergoing degenerative changes. $e$ The bulbous swelling of the hair shaft is located close to the orifice. The cortex of the hair shaft is fragmented and broken hair shaft fragments are oriented horizontally. f Histology of a feline control hair follicle. $\mathbf{g}$ Trichogram of a feline control hair
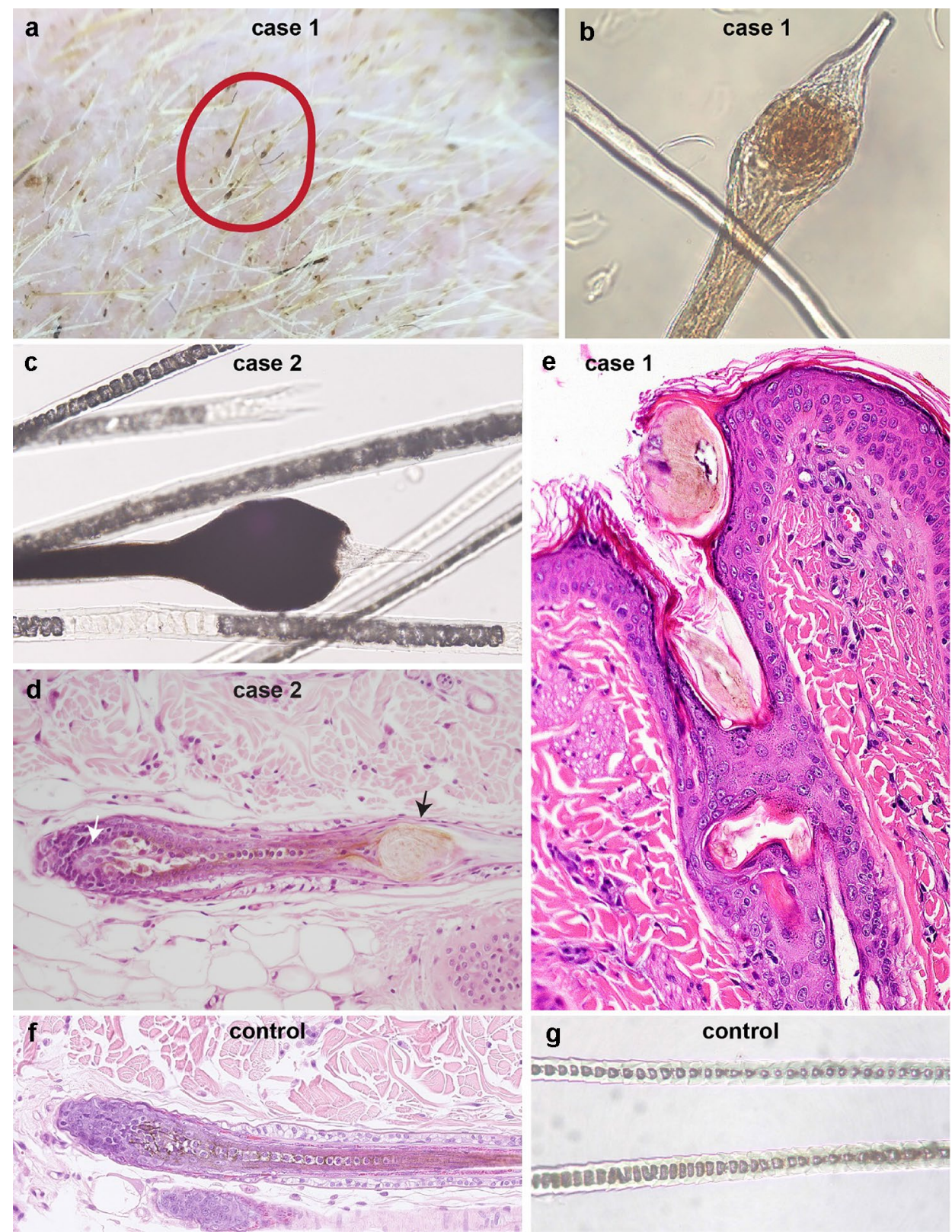

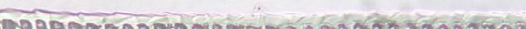

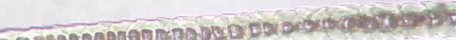

bulbous swellings. These swellings were present anywhere in the hair shaft but most often close to the tip (Fig. 2a, b).

In 2010, a 1.5 year old male castrated DSH cat (case no. 2) from a local shelter in Germany was presented with progressive alopecia of the dorsum, the plantar, and palmar surfaces of the limbs, the convex pinnae, and most of the face, as previously published (Fig. 1c, d) (Rostaher et al. 2021). In addition to body hairs being affected, the vibrissae were short and broken. Macroscopic evaluation of the skin surface and microscopic examination of trichograms revealed many short, broken hair shafts, some of which had bulbous or lance-head shaped ends (Fig. 2c). All of the cat's littermates showed similar skin lesions.
The histopathological evaluation revealed that the number and size of the hair follicles was normal and the presence of hair shafts in all hair follicles. Most hair follicles were in anagen. Numerous, but not all hair shafts were dystrophic. The dystrophy was characterized by severe well circumscribed thickening of the hair shafts starting above the melanogenic zone (Fig. 2d). In addition, there was loss of a ladder-like pattern of pigment distribution. In Fig. 2e, the swelling of the hair shaft was present more distally in the infundibulum. This reflects most likely the outgrowing of the bulbous swelling and represents the lance-head shaped hair tip. Additionally, the dystrophy was characterized by an irregular outer contour of the hair shafts, fragmentation 
Table 2 Results of variant filtering in the affected cats against 61 control genomes

\begin{tabular}{lcc}
\hline Filtering step & Variants case no. 1 & Variants case no. 2 \\
\hline All variants & $5,955,464$ & $6,162,272$ \\
Private variants & 26,179 & 26,624 \\
$\begin{array}{l}\text { Protein-changing } \\
\text { private variants }\end{array}$ & 76 & 93 \\
$\begin{array}{l}\text { Protein-changing } \\
\text { private variants in } \\
D S G 4\end{array}$ & 1 & \\
\hline
\end{tabular}

Only homozygous variants are reported

within the cortex and the cuticle, and dense eosinophilic cornified material that surrounded the hair shafts. In some infundibula, the fragmented hair shafts were oriented horizontally. Furunculosis was present in one biopsy. Sebaceous and sweat glands were normal. The epidermis was moderately hyperplastic and covered by a small amount of laminar orthokeratotic keratin (Fig. 2e).

\section{Genetic analysis}

Given the results of the clinical and histopathological analysis, DSG4 was considered the primary functional candidate gene for the observed phenotype in both cases. To characterize the underlying causative genetic variant, we sequenced the genome of the affected cats and searched for private variants in DSG4. Since the cats were not known to be related, we hypothesized that their phenotypes were due to independent pathogenic variants. We therefore searched for private variants in each of the affected cats' genomes individually by comparing them to the genomes of 61 other cats (Table 2, Table S1, Table S2).

This analysis identified a single homozygous private protein-changing candidate variant in DSG4 in each of the cases. The two variants can be designated as ChrD3:55,315,010del (FelCat9.0) for case no. 1 and ChrD3:55,336,127del (FelCat9.0) for case no. 2. Both variants represented $1 \mathrm{bp}$ deletions, causing frameshifts and resulting in premature stop codons (Table 3 ). The variants truncate $98 \%$ and $43 \%$ of the open-reading frame, respectively.

We confirmed the presence of the DSG4 frameshift variants by Sanger sequencing (Fig. 3) and genotyped a cohort of 46 unaffected cats from different breeds for both variants. The affected cats carried one frameshift allele in a homozygous state and were homozygous wild-type at the variant of the other case. All other non-affected cats were homozygous for the wild-type allele at both variants (Table 4).

Table 3 Variant designations of the identified DSG4 frameshift deletions

\begin{tabular}{llll}
\hline Cats & \multicolumn{2}{l}{ HGVS variant designations } & \\
\cline { 2 - 4 } & Genomic (FelCat9.0) & $\begin{array}{l}\text { mRNA } \\
\text { (XM_019815116.1) }\end{array}$ & Protein (XP_019670675.1) \\
\hline Case no. 1 & ChrD3:55,315,010del & c.76del & p.Ile26Leufs*4 \\
Case no. 2 & ChrD3:55,336,127del & c.1777del & p.His593Thrf*23 \\
\hline
\end{tabular}

a

$$
\frac{{ }^{25} \mathrm{Phe}}{\mathrm{T} \text { T T A T T G T T G A G }} \frac{{ }^{26} \text { lle }}{{ }^{27} \text { Val }} \frac{{ }^{28} \mathrm{Glu}}{{ }^{2} \mathrm{~A}}
$$

wildtype $w t / w t$

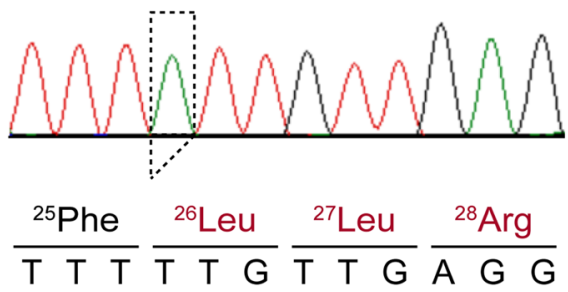

\section{affected} case no. 1 del/del

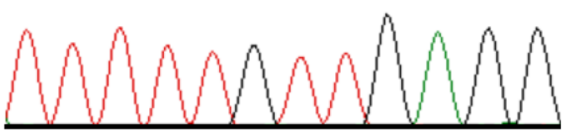

b $\frac{{ }^{592} \mathrm{Asn}}{\mathrm{A} \mathrm{A} \mathrm{C}} \frac{{ }^{593} \mathrm{His}}{\mathrm{C} \mathrm{A} \mathrm{C}} \frac{{ }^{594} \mathrm{lle}}{\text { A T A T G T }}$ wildtype
$w t / w t$

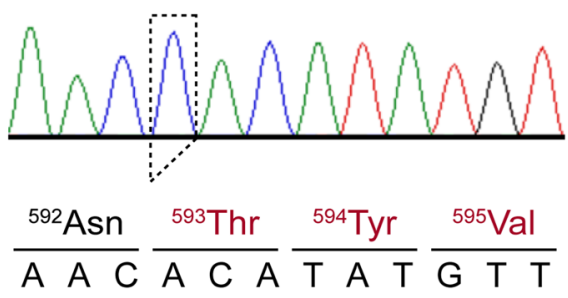

affected case no. 2 $\mathrm{del} / \mathrm{del}$

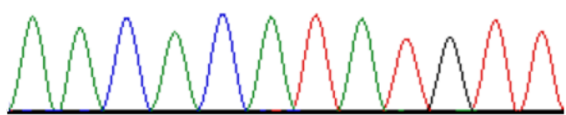

Fig. 3 Details of the a DSG4:c.76del and b DSG4:c.1777del variants. Representative electropherograms of a control and the affected cats are shown. The amino acid translations of the wild-type and mutant alleles are indicated 
Table 4 Genotype-phenotype association of the DSG4 variants with hair shaft dystrophy in cats

\begin{tabular}{|c|c|c|c|c|c|c|}
\hline \multirow[t]{2}{*}{ Cats } & \multicolumn{3}{|c|}{ c.76del } & \multicolumn{3}{|c|}{ c.1777del } \\
\hline & $\mathrm{wt} / \mathrm{wt}$ & wt/del & $\mathrm{del} / \mathrm{del}$ & $\mathrm{wt} / \mathrm{wt}$ & wt/del & $\mathrm{del} / \mathrm{del}$ \\
\hline Case no. 1 & - & - & 1 & 1 & - & - \\
\hline Case no. 2 & 1 & - & - & - & - & 1 \\
\hline Controls $(n=46)$ & 46 & - & - & 46 & - & - \\
\hline
\end{tabular}

\section{Discussion}

In this study, we investigated two unrelated DSH cats affected with hair shaft dystrophy. One of the two investigated cats (case no. 2) was part of a previous study about the clinical, histological, and ultrastructural features of the disease, which revealed significant similarity to $D s g 4^{l a h}$ and Dsg $4^{\text {lahJ }}$ mutant mice (Rostaher et al. 2021). The other case (case no. 1) was an unrelated DSH cat that presented with a similar clinical and histopathological phenotype.

We sequenced the genomes of both cats and filtered for private homozygous variants in our primary functional candidate gene $D S G 4$. This analysis identified independent private variants in each of the cases. Both variants represented frameshift deletions predicted to result in true null alleles. The finding of independent loss-of-function variants in the same gene in cats with comparable phenotype represents a very strong evidence for the causality of these variants.

$D S G 4$ has been identified as the fourth and last member of the desmoglein family (Whittock and Bower 2003). In human anagen hair follicles, DSG4 is expressed in the precortex, cortex, and lower cuticle of the hair shaft. It is additionally expressed in the inner root sheath cuticle above the bulb region (Bazzi et al. 2006). As component of the desmosomes, DSG4 is involved in linking the intracellular network of keratins of a trichocyte to the intermediate filaments of the neighbouring trichocytes. This intra- and inter-cellular network of keratins and desmosomes provides the hair shaft with its tensile strength and elasticity (Bazzi et al. 2009). It also plays a central role in coordinating cellular dynamics in the lower hair follicle during the switch from proliferation to differentiation (Kljuic et al. 2003). The mutant cats had a prominent hair shaft defect characterized by a bulbous swelling distal to the melanogenic zone. This probably leads to an increased fragility and the formation of a lance-head shaped hair tip (Rostaher et al. 2021).

Variants in DSG4 have previously been described in mice and rats with lanceolate hair phenotype, and in human patients with localized autosomal recessive hypotrichosis, as well as monilethrix-like hypotrichosis (Kljuic et al. 2003; Jahoda et al. 2004; Moss et al. 2004; Nagasaka et al. 2004; Rafiq et al. 2004; Messenger et al. 2005; John et al. 2006; Schaffer et al. 2006; Shimomura et al. 2006; Zlotogorski et al. 2006; Wajid et al. 2007). We assume that loss of DSG4 function leads to an incorrect formation of the desmosomes resulting in impaired adhesion between neighbouring trichocytes and a defective hair shaft formation in the affected cats.

In summary, we identified two likely disease-causing variants in DSG4 in cats with a recessive hair shaft dystrophy. The hair phenotype of the affected cats was comparable to the phenotype in lanceolate hair mice. Our findings therefore corroborate the relevance of desmoglein 4 for correct hair shaft formation and the known genotype-phenotype correlations from other species. The affected cats were homozygous for the mutant alleles, indicating that inbreeding contributed to the observed genodermatoses. To the best of our knowledge, this study represents the first report of pathogenic $D S G 4$ variants in domestic animals.

Supplementary Information The online version contains supplementary material available at https://doi.org/10.1007/s00438-021-01842-6.

Acknowledgements The authors are grateful to all cat owners who donated samples and shared health and pedigree data of their cats. We thank the Next Generation Sequencing Platform of the University of Bern for performing the high-throughput sequencing experiments, and the Interfaculty Bioinformatics Unit of the University of Bern for providing high-performance computing infrastructure.

Author contributions Conceptualization: TL and AR; investigation: SK, AR, SR, and MW; data curation: VJ; writing - original draft: SK, AR, SR, MW, and TL; writing-review and editing: SK, AR, SR, VJ, JS, MW, and TL; supervision: TL. All authors have read and agreed to the published version of the manuscript.

Funding This research was funded by the Swiss National Science Foundation, Grant Number 310030_200354.

Availability of data and materials The genome sequence data were submitted to the European Nucleotide Archive (ENA). All accession numbers are listed in Table $\mathrm{S} 1$.

Code availability Not applicable.

\section{Declarations}

Conflict of interest The authors declare that they have no conflict of interest.

Ethics approval The affected cats in this study were privately owned and skin biopsies and blood samples for diagnostic purposes were collected with the consent of their owners. The collection of blood samples from healthy control cats was approved by the "Cantonal Committee for Animal Experiments" (Canton of Bern; permit 71/19). 
Consent to participate Not applicable.

Consent for publication Not applicable.

Open Access This article is licensed under a Creative Commons Attribution 4.0 International License, which permits use, sharing, adaptation, distribution and reproduction in any medium or format, as long as you give appropriate credit to the original author(s) and the source, provide a link to the Creative Commons licence, and indicate if changes were made. The images or other third party material in this article are included in the article's Creative Commons licence, unless indicated otherwise in a credit line to the material. If material is not included in the article's Creative Commons licence and your intended use is not permitted by statutory regulation or exceeds the permitted use, you will need to obtain permission directly from the copyright holder. To view a copy of this licence, visit http://creativecommons.org/licenses/by/4.0/.

\section{References}

Ahmed A, Almohanna H, Griggs J, Tosti A (2019) Genetic hair disorders: a review. Dermatol Ther (heidelb) 9:421-448. https://doi. org/10.1007/s13555-019-0313-2

Bazzi H, Getz A, Mahoney MG, Ishida-Yamamoto A, Langbein L, Wahl JK 3rd, Christiano AM (2006) Desmoglein 4 is expressed in highly differentiated keratinocytes and trichocytes in human epidermis and hair follicle. Differentiation 74:129-140. https:// doi.org/10.1111/j.1432-0436.2006.00061.x

Bazzi H, Demehri S, Potter CS, Barber AG, Awgulewitsch A, Kopan $\mathrm{R}$, Christiano AM (2009) Desmoglein 4 is regulated by transcription factors implicated in hair shaft differentiation. Differentiation 78:292-300. https://doi.org/10.1016/j.diff.2009.06.004

Chen J, Jaeger K, Den Z, Koch PJ, Sundberg JP, Roop DR (2008) Mice expressing a mutant Krt75 (K6hf) allele develop hair and nail defects resembling pachyonychia congenita. J Invest Dermatol 128:270-279. https://doi.org/10.1038/sj.jid.5701038

Cheng AS, Bayliss SJ (2008) The genetics of hair shaft disorders. J Am Acad Dermatol 59:1-22. https://doi.org/10.1016/j.jaad.2008. 04.002

Cingolani P, Platts A, le Wang L, Coon M, Nguyen T, Wang L, Land SJ, Lu X, Ruden DM (2012) A program for annotating and predicting the effects of single nucleotide polymorphisms, SnpEff: SNPs in the genome of Drosophila melanogaster strain w1118; iso-2; iso-3. Fly (austin) 6:80-92. https://doi.org/10.4161/fly. 19695

Jagannathan V, Drögemüller C, Leeb T, Dog Biomedical Variant Database Consortium (DBVDC) (2019) A comprehensive biomedical variant catalogue based on whole genome sequences of $582 \mathrm{dogs}$ and eight wolves. Anim Genet 50:695-704. https://doi.org/10. 1111/age. 12834

Jahoda CA, Kljuic A, O'Shaughnessy R, Crossley N, Whitehouse CJ, Robinson M, Reynolds AJ, Demarchez M, Porter RM, Shapiro L, Christiano AM (2004) The lanceolate hair rat phenotype results from a missense mutation in a calcium coordinating site of the desmoglein 4 gene. Genomics 83:747-756. https://doi.org/10. 1016/j.ygeno.2003.11.015

John P, Tariq M, Arshad Rafiq M, Amin-Ud-Din M, Muhammad D, Waheed I, Ansar M, Ahmad W (2006) Recurrent intragenic deletion mutation in desmoglein 4 gene underlies autosomal recessive hypotrichosis in two Pakistani families of Balochi and Sindhi origins. Arch Dermatol Res 298:135-137. https://doi.org/10.1007/ s00403-006-0671-3
Kljuic A, Bazzi H, Sundberg JP, Martinez-Mir A, O’Shaughnessy R, Mahoney MG, Levy M, Montagutelli X, Ahmad W, Aita VM, Gordon D, Uitto J, Whiting D, Ott J, Fischer S, Gilliam TC, Jahoda CA, Morris RJ, Panteleyev AA, Nguyen VT, Christiano AM (2003) Desmoglein 4 in hair follicle differentiation and epidermal adhesion: evidence from inherited hypotrichosis and acquired pemphigus vulgaris. Cell 113:249-260. https://doi.org/ 10.1016/S0092-8674(03)00273-3

Messenger AG, Bazzi H, Parslew R, Shapiro L, Christiano AM (2005) A missense mutation in the cadherin interaction site of the desmoglein 4 gene underlies localized autosomal recessive hypotrichosis. J Invest Dermatol 125:1077-1079. https://doi.org/10.1111/j. 0022-202X.2005.23903.x

Montagutelli X, Hogan ME, Aubin G, Lalouette A, Guénet JL, King LE, Sundberg JP (1996) Lanceolate hair (lah): a recessive mouse mutation with alopecia and abnormal hair. J Invest Dermatol 107:20-25. https://doi.org/10.1111/1523-1747.ep12297438

Moss C, Martinez-Mir A, Lam H, Tadin-Strapps M, Kljuic A, Christiano AM (2004) A recurrent intragenic deletion in the desmoglein 4 gene underlies localized autosomal recessive hypotrichosis. J Invest Dermatol 123:607-610. https://doi.org/10.1111/j.0022202X.2004.23311.x

Nagasaka T, Nishifuji K, Ota T, Whittock NV, Amagai M (2004) Defining the pathogenic involvement of desmoglein 4 in pemphigus and staphylococcal scalded skin syndrome. J Clin Invest 114:14841492. https://doi.org/10.1172/JCI20480

Nakamura M, Sundberg JP, Paus R (2001) Mutant laboratory mice with abnormalities in hair follicle morphogenesis, cycling, and/ or structure: annotated tables. Exp Dermatol 10:369-390. https:// doi.org/10.1034/j.1600-0625.2001.100601.x

Rafiq MA, Ansar M, Mahmood S, Haque S, Faiyaz-ul-Haque M, Leal SM, Ahmad W (2004) A recurrent intragenic deletion mutation in DSG4 gene in three Pakistani families with autosomal recessive hypotrichosis. J Invest Dermatol 123:247-248. https://doi.org/10. 1111/j.0022-202X.2004.22715.x

Rostaher A, Bettenay S, Specht L, Silva KA, Bechtold L, Chen J, Majzoub M, Mueller RS, Sundberg JP (2021) Hair follicle dystrophy in a litter of domestic cats resembling lanceolate hair mutant mice. Vet Dermatol 32:74-e14. https://doi.org/10.1111/vde.12925

Schaffer JV, Bazzi H, Vitebsky A, Witkiewicz A, Kovich OI, Kamino H, Shapiro LS, Amin SP, Orlow SJ, Christiano AM (2006) Mutations in the desmoglein 4 gene underlie localized autosomal recessive hypotrichosis with monilethrix hairs and congenital scalp erosions. J Invest Dermatol 126:1286-1291. https://doi.org/10. 1038/sj.jid.5700237

Schneider MR, Schmidt-Ullrich R, Paus R (2009) The hair follicle as a dynamic miniorgan. Curr Biol 19:R132-142. https://doi.org/10. 1016/j.cub.2008.12.005

Shimomura Y, Christiano AM (2010) Biology and genetics of hair. Annu Rev Genomics Hum Genet 11:109-132. https://doi.org/10. 1146/annurev-genom-021610-131501

Shimomura Y, Sakamoto F, Kariya N, Matsunaga K, Ito M (2006) Mutations in the desmoglein 4 gene are associated with monilethrix-like congenital hypotrichosis. J Invest Dermatol 126:12811285. https://doi.org/10.1038/sj.jid.5700113

Sundberg JP (1994) Handbook of mouse mutations with skin and hair abnormalities: animal models and biomedical tools. CRC Press, Boca Raton

Sundberg JP, King LE (1996) Mouse mutations as animal models and biomedical tools for dermatological research. J Invest Dermatol 106:368-376. https://doi.org/10.1111/1523-1747.ep12343152

Sundberg JP, King LE (2000) Skin and its appendages: normal anatomy and pathology of spontaneous, transgenic and targeted mouse mutations. In: Ward JM, Mahler JF, Maronpot RR, Sundberg JP (eds) Pathology of genetically engineered mice. Iowa State University Press, Ames, pp 181-213 
Sundberg JP, Boggess D, Bascom C, Limberg BJ, Shultz LD, Sundberg BA, King LE, Montagutelli X (2000) Lanceolate hair-J (lahJ): a mouse model for human hair disorders. Exp Dermatol 9:206-218. https://doi.org/10.1034/j.1600-0625.2000.009003206.x

Wajid M, Bazzi H, Rockey J, Lubetkin J, Zlotogorski A, Christiano AM (2007) Localized autosomal recessive hypotrichosis due to a frameshift mutation in the desmoglein 4 gene exhibits extensive phenotypic variability within a Pakistani family. J Invest Dermatol 127:1779-1782. https://doi.org/10.1038/sj.jid.5700791

Welle MM, Wiener DJ (2016) The hair follicle: a comparative review of canine hair follicle anatomy and physiology. Toxicol Pathol 44:564-574. https://doi.org/10.1177/0192623316631843

Whittock NV, Bower C (2003) Genetic evidence for a novel human desmosomal cadherin, desmoglein 4. J Invest Dermatol 120:523-530. https://doi.org/10.1046/j.1523-1747.2003.12113.x

Wiener DJ, Groch KR, Brunner MAT, Leeb T, Jagannathan V, Welle MM (2020) Transcriptome profiling and differential gene expression in canine microdissected anagen and telogen hair follicles and interfollicular epidermis. Genes (basel) 11:884. https:// doi.org/10.3390/genes11080884

Zlotogorski A, Marek D, Horev L, Abu A, Ben-Amitai D, Gerad L, Ingber A, Frydman M, Reznik-Wolf H, Vardy DA, Pras E (2006) An autosomal recessive form of monilethrix is caused by mutations in DSG4: clinical overlap with localized autosomal recessive hypotrichosis. J Invest Dermatol 126:1292-1296. https://doi.org/ 10.1038/sj.jid.5700251

Publisher's Note Springer Nature remains neutral with regard to jurisdictional claims in published maps and institutional affiliations. 